la revue La revue pour l'histoire du CNRS

POUR LHISTORE DU CNRS $\quad 26 \mid 2010$

Sport, recherche et société

\title{
Le budget du CNRS de 1969 à 2000.
}

Bruno Marnot

\section{(2) OpenEdition}

Journals

Édition électronique

URL : https://journals.openedition.org/histoire-cnrs/9273

DOI : 10.4000/histoire-cnrs.9273

ISSN : 1955-2408

Éditeur

CNRS Éditions

Édition imprimée

Date de publication : 30 novembre 2010

Pagination : 36-41

ISBN : 978-2-271-07145-3

ISSN : 1298-9800

Référence électronique

Bruno Marnot, "Le budget du CNRS de 1969 à 2000. », La revue pour l'histoire du CNRS [En ligne], 26 | 2010, mis en ligne le 24 février 2013, consulté le 20 mai 2021. URL : http://journals.openedition.org/ histoire-cnrs/9273; DOI : https://doi.org/10.4000/histoire-cnrs.9273

Ce document a été généré automatiquement le 20 mai 2021.

Comité pour l'histoire du CNRS 


\section{Le budget du CNRS de 1969 à 2000.}

\section{Bruno Marnot}

1 La période qui couvre les années 1970 est sans doute l'une des plus délicates à interpréter sur le plan financier, en grande partie à cause des récriminations contemporaines contre les restrictions budgétaires qui n'ont pas toujours contribué à éclairer le débat. L'historien dispose néanmoins d'une documentation plus abondante à partir de cette période, qui tend même à devenir pléthorique au fil des années et ne permet pas toujours, en définitive, de mieux instruire son jugement ${ }^{1}$.

\section{La fin des budgets de croissance (1969-1980)}

2 L'évolution budgétaire du CNRS des années 1970 s'inscrit dans un contexte général marqué par le ralentissement de la croissance des crédits publics, sensible dès les années 1967-1968. L'effort public de recherche-développement (R-D) de la France augmente moins vite entre 1970 et 1977 que celui des autres pays de la CEE. Si l'Europe des neuf affiche en moyenne 2,3\% de variation des crédits de R-D, la France occupe, loin derrière ses partenaires, la dernière position avec $0,8 \%$. Dans ce contexte préoccupant, le CNRS parvient néanmoins, à l'inverse de la décennie précédente, à améliorer sa position relative. De 1970 à 1977, le volume de l'établissement passe de $5,7 \%$ à 7,7 \% de la dépense intérieure de recherche-développement (DIRD). De manière plus précise, si son budget représente, en 1970, 12,9\% de la DIRD exécutée par les administrations, il s'établit à hauteur de $20 \%$ en 1977. À cette date, son poids financier représente un quart des crédits totaux de l'enveloppe recherche ${ }^{2}$.

3 Aussi, contrairement à maints propos alarmistes de l'époque, qui s'élèvent notamment au sein de la communauté scientifique, les années 1970 ne se soldent pas par une diminution relative et encore moins absolue du budget du CNRS. Entre 1971 et 1981, celui-ci augmente de $71 \%$ en francs constants, contre, il est vrai, $169 \%$ de 1961 à 1971. Le budget continue, par conséquent, de croître mais à un rythme bien inférieur. Le dessin de sa courbe ne présente plus la belle trajectoire ascensionnelle de la décennie précédente. Le CNRS est bien entré dans une phase de croissance budgétaire molle. 
Dans ce contexte quelque peu morose, la part des crédits d'équipement se rétablit légèrement, passant de 20,3\% en 1969 du budget total à 24,4\% en 1980. Cette amélioration est moins imputable à leur progression intrinsèque qu'à l'affaissement du rythme des dépenses de fonctionnement, qui est passé de + $253 \%$ entre 1959 et 1968 à + $70 \%$ entre 1969 et 1980. Malgré tout, les autorisations de programme souffrent toujours d'irrégularité, véritable maladie chronique du CNRS, suffisante en soi pour indisposer la communauté scientifique qui ne sait jamais de quoi le lendemain sera fait. Or, les moyens mis à la disposition de la recherche deviennent véritablement problématiques au cours des années 1970. Ceux-ci se répartissent de la manière suivante selon les différents secteurs scientifiques du CNRS.

\section{RÉPARTITION SECTORIELLE EN \% DU BUDGET TOTAL EN 1971}

\begin{tabular}{lc}
\hline Physique nucléaire & 12,4 \\
\hline Physique non nucléaire & 14,7 \\
\hline Chimie & 13,7 \\
\hline $\begin{array}{l}\text { Sciences de la Terre, de I'océan, } \\
\text { de I'atmosphère et de I'espace (TOAE) }\end{array}$ & 8,37 \\
\hline Sciences de la Vie & 23,3 \\
\hline Sciences de l'Homme & 11,4 \\
\hline Mathématiques et informatique & 1,87
\end{tabular}

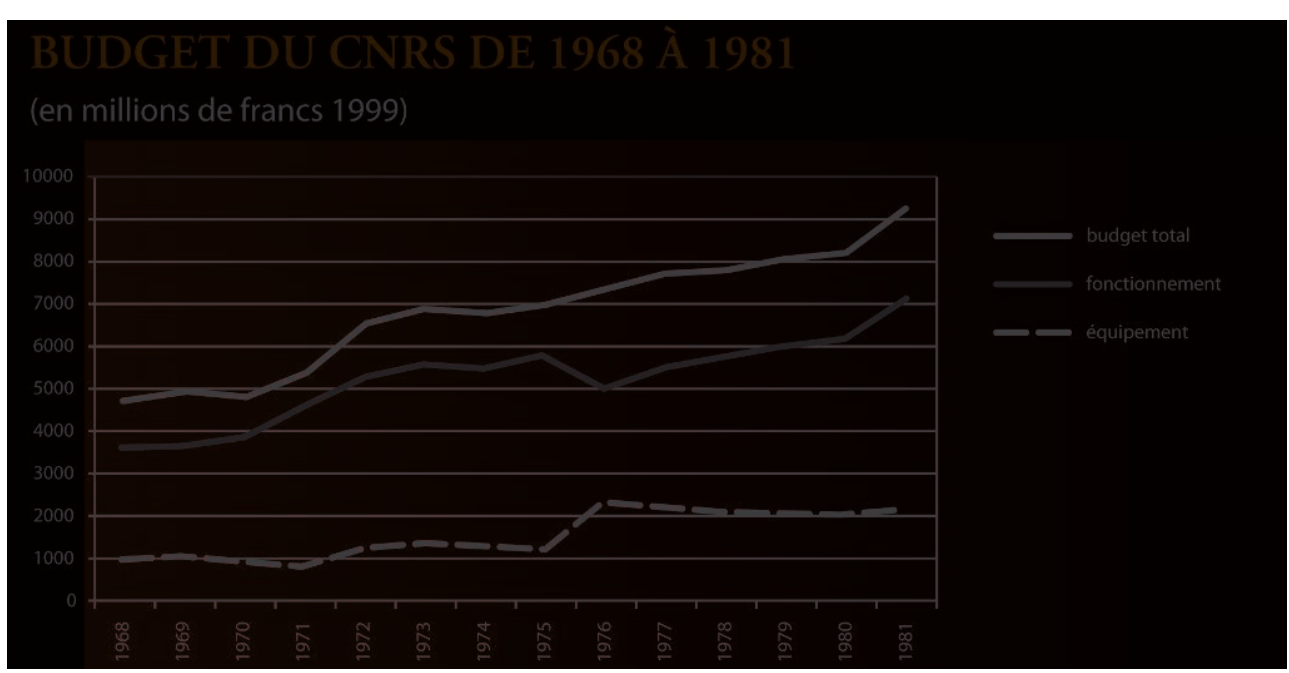

5 L'étude comparative de ces séries montre un respect relatif des équilibres sectoriels tout au long de la décennie. Le vaste domaine de la physique ne permet pas d'établir des comparaisons directes en raison de son redécoupage interne à la suite de la création de l'Institut national de physique nucléaire et de physique des particules (IN2P3). Le même constat vaut pour les sciences de l'environnement partagées en 1980 entreles Sciences de la Terre, de l'océan, de l'atmosphère et de l'espace (TOAE) et l'Institut national d'astronomie et de géophysique (Inag). Ces dernières augmentent leur poids relatif au cours de la décennie, alors que les sciences de la vie enregistrent un recul sensible. Par ailleurs, la rapide montée en puissance des instituts nationaux est visible, en raison de la part croissante qu'ils occupent dans le budget total du CNRS jusqu'en 1974 où ils culminent à $15 \%$; par la suite, cette part subit une érosion régulière pour se situer à hauteur de $12 \%$ au début des années 1980. Les instituts 
nationaux n'échappent pas à la chute globale en volume des gros équipements qui se produit entre 1974 et 1979. Seules les sciences humaines augmentent leur volume de $80 \%$ entre ces deux dates, mais il faut préciser que le niveau d'équipement de ce dernier secteur était extrêmement faible avant 1974. Cette évolution inquiétante est une autre manière de montrer que les moyens mis à la disposition des laboratoires tendent à se dégrader au CNRS.

6 L'analyse de la structure du budget de fonctionnement permet de mieux apprécier la situation vicieuse qui se développe dans les finances de l'institution. À défaut d'être nouvelle, celle-ci demeurait indolore en période d'abondance budgétaire, alors qu'elle se révèle avec acuité au moment où la manne financière se tarit. En effet, les dépenses de personnel augmentent à nouveau de manière significative dès 1969 (74,7\%) pour atteindre un maximum de 97,6\% en 1980 ! Autant dire, la quasi-totalité du budget de fonctionnement, ce qui réduit à néant les dépenses de fonctionnement proprement dites. Les proportions gigantesques prises par la masse salariale deviennent tout aussi inquiétantes lorsqu'on la rapporte au budget total : 55,5\% de celui-ci en 1969 mais pratiquement les trois-quarts en 1980.

7 La pression croissante de la masse salariale s'explique par la conjugaison de trois phénomènes structurels de l'organisme, à la fois démographiques et professionnels : l'accroissement de l'effectif, le vieillissement du corps des chercheurs, la progression de l'indice salarial. En effet, de 1970 à 1980, l'effectif total du CNRS augmente de 43,8 \%. Le Centre favorise davantage le recrutement des $\operatorname{ITA}^{3}(+48,8 \%)$ que celui des chercheurs $(+36,6 \%)$, autant pour améliorer l'encadrement technique des laboratoires que pour limiter les effets financiers liés à l'embauche de nouveaux chercheurs. Cette politique qui consiste à limiter le recrutement du personnel scientifique à partir de 1973 est la contrepartie de la priorité accordée par la nouvelle direction à l'amélioration des moyens mis à la disposition de la recherche. La politique de limitation du recrutement contient cependant un effet pervers, qui est le vieillissement automatique de la population du Centre. Cette évolution rejaillit sur le poids plus élevé de l'indice salarial: une main d'œuvre qualifiée vieillissante coûte plus cher à son employeur. La rationalisation des choix budgétaires voulue par Pierre Creyssel a pour but de sortir le Centre de sa progressive paralysie financière, en même temps qu'elle doit répondre au concept nouveau « d'entreprise de sciences ».

\section{De l'éphémère relance à l'asphyxie financière (1981-2000)}

8 En 1981, la gauche au pouvoir a pour ambition de relancer la politique de la recherche. Celle-ci trouve une traduction financière immédiate : le budget total de l'établissement augmente, en francs constants, de $12,3 \%$ en 1981, puis de $11,7 \%$ en 1982 et de $6,3 \%$ encore l'année suivante. Ces chiffres s'affichent d'emblée comme les trois plus forts taux de croissance des deux dernières décennies du siècle, à l'exception notable de l'année 1995 dont l'augmentation de 18,6 \% trahit en fait plus une situation de crise que de relance. Toutefois, la relance budgétaire dont bénéficie le CNRS au tout début des années 1980 ne résiste pas à la conjoncture économique et à la réduction consécutive des marges de manœuvre. En ces décennies de faible croissance, celle du budget annuel moyen du CNRS continue à s'exténuer : 3,14\% par an entre 1981 et 1990, puis 2,7\% entre 1991 et 1999. Cette évolution explique que le CNRS ait de plus en plus tendance à 
puiser dans ses propres fonds au cours de cette période. En effet, le ratio ressources propres / subventions de l'État passe de 1,6 en 1981 à 11,5 en 1999.

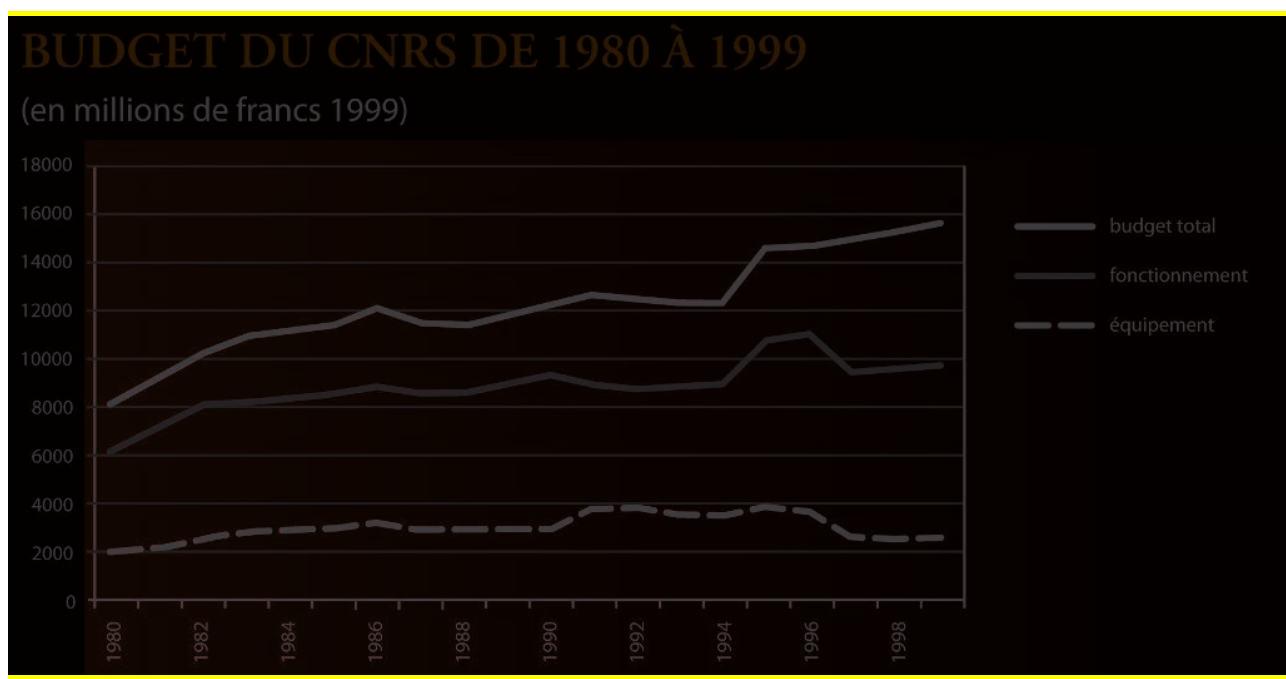

9 À l'instar de la décennie précédente, le CNRS ne semble pas avoir pâti de cette faible croissance budgétaire au sein de la recherche civile et publique française. Sa part passe de $24,3 \%$ en 1981 à $26 \%$ en 1999 dans le total du budget civil de recherche et développement $(\mathrm{BCRD})^{4}$. L'organisme maintient par conséquent ses positions sur l'ensemble des vingt dernières années du siècle. En 1994, le budget consolidé du CNRS représente $63 \%$ - soit presque les deux tiers - du budget des établissements publics scientifiques et techniques (EPST).

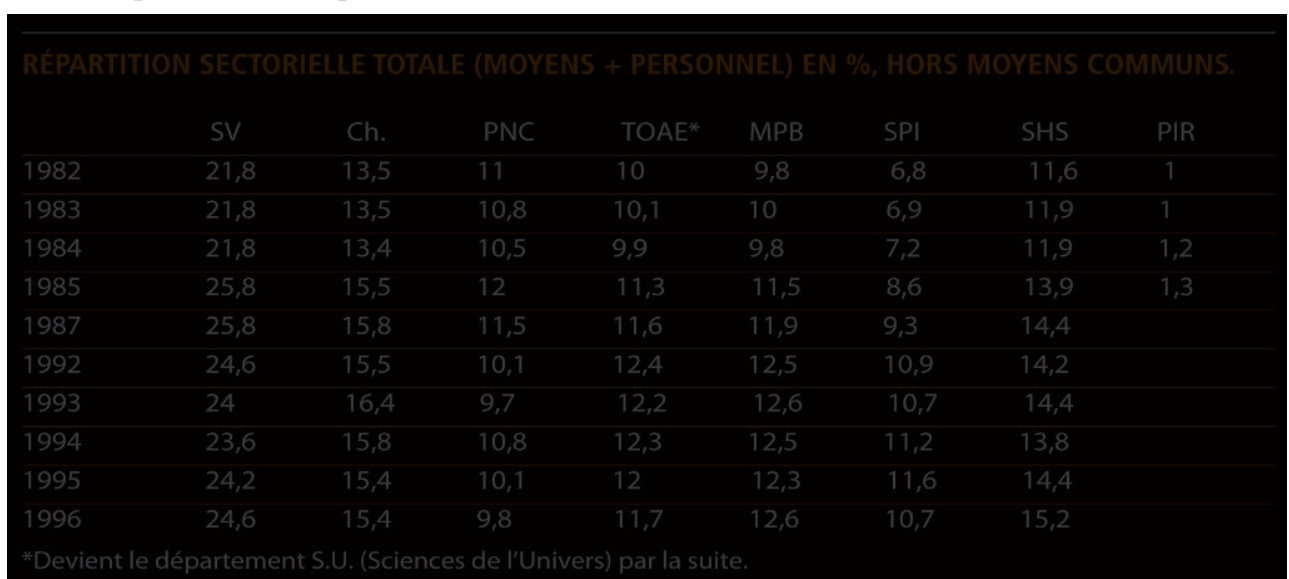

La répartition sectorielle des moyens souligne quelques évolutions intéressantes. Trois groupes se dégagent. Le premier comprend les Sciences physiques pour l'ingénieur (SPI) et les Sciences humaines et sociales (SHS), qui connaissent une augmentation substantielle de leurs moyens entre 1982 et 1996. La montée en puissance des SPI rappelle que le développement des sciences de transfert et de la politique de valorisation au cours des années 1970, non seulement se poursuit au cours des décennies suivantes mais devient une priorité affichée de la politique scientifique de l'organisme. Le département SHS bénéficie toujours, quant à lui, de l'effort de rattrapage antérieur en raison du nouveau rôle assigné aux sciences humaines dans la connaissance de l'environnement. Il représente le troisième département du CNRS par ordre de moyens totaux. Les deux secteurs lourds que sont les Sciences de la Vie (SV) et la Chimie connaissent, en revanche, une croissance plus modérée. Si les SV constituent, 
avec les SPI, la deuxième priorité des années 1980, cette évolution ne se confirme pas au cours de la décennie suivante. Le département des Mathématiques et physique de base (MPB) et celui des Sciences de l'Univers (ex-TOAE) subissent une stagnation identique au cours des années 1990. Enfin, la Physique nucléaire et corpusculaire enregistre un léger recul sur l'ensemble de la période, même si dans le détail l'évolution budgétaire de ce département demeure assez aléatoire.

11 Ces départements ont-ils connu une amélioration de leurs moyens? En fait, la disproportion entre équipement et fonctionnement est loin de se résorber au cours de cette période. La part de l'équipement dans le budget total passe de $24 \%$ à $16,1 \%$ entre 1982 et 1999. À une première phase de consolidation autour du quart du budget total, succède un léger mais irrésistible glissement de 1989 à 1991. Un pic historique de $30 \%$ est certes atteint en 1992, mais il est suivi d'une nouvelle érosion jusqu'à la fin de la décennie.

12 La progressive sclérose budgétaire du CNRS peut, en premier lieu, être identifiée dans le très net ralentissement de la progression des dépenses ordinaires et des autorisations de programme par rapport à la décennie précédente : $+30,7 \%$ pour les premières entre 1981 et 1990 contre $+70 \%$ auparavant, $+38,2 \%$ pour les secondes contre $97 \%$. La structure des crédits de fonctionnement demeure le talon d'Achille budgétaire du Centre. Les dépenses de personnel atteignent, en effet, 84,4\% des dépenses ordinaires en 1987. Le décret du $1^{\mathrm{er}}$ mars 1984 modifie d'ailleurs la nomenclature du budget du CNRS pour rendre mieux compte de sa réalité. Celui-ci est désormais présenté en trois sections. La première regroupe l'ensemble des crédits alloués aux dépenses de personnel ; la deuxième correspond aux dépenses ordinaires et à une réserve générale; la troisième section comprend une dotation globale destinée aux unités de recherche et des crédits pour opérations programmées (actions d'intervention sur programme et actions incitatives). Le budget total n'en demeure pas moins grevé par le premier poste.

13 À la différence des années 1960 , le poids structurel et croissant des dépenses de personnel ne s'explique plus par la politique de recrutement massif. La décélération du recrutement est manifeste dès la deuxième moitié des années $1980:+1,8 \%$ entre 1986 et 1991 contre $+2,6 \%$ entre 1981 et 1986 pour les chercheurs, tandis que les chiffres relatifs au recrutement des ITA avoisinent l'insignifiance avec respectivement $+0,2 \%$ contre $+0,7 \%$ dans les mêmes tranches chronologiques. La pyramide des âges de l'ensemble du personnel fournit, avec plus d'éclat qu'au cours des années 1970, une autre clé du problème. La tranche d'âge la plus importante passe de 30-39 ans en 1977 à 40-49 ans en 1990, puis à 45-54 ans en 1994. L'âge moyen des chercheurs augmente de 4,8 ans entre 1980 et 1994 et de 5,5 ans pour les ITA. Faute de renouvellement substantiel, la population du CNRS vieillit de manière inéluctable, phénomène qui conduit à renforcer des effets pervers constatés dès les années 1970.

14 Les schémas directeurs disponibles à partir des années 1980, préparés dans le cadre des plans triennaux par la Direction de la stratégie et des programmes, fournissent de précieuses analyses qui permettent de discerner les vices de la structure budgétaire du CNRS. Deux sources de déséquilibre sont invariablement rappelées. La première concerne évidemment les crédits relatifs au personnel qui pèsent toujours plus sur les moyens mis au service de la recherche. Pour contrecarrer cette évolution, le schéma de 1984-1986 enjoint de "préserver un flux régulier et suffisamment soutenu de recrutement de jeunes chercheurs ", alors que la communauté scientifique est entrée 
dans une logique durable de fonctionnarisation et d'intégration des hors-statuts. Cette politique est à l'œuvre au commencement des années 1980, notamment grâce à l'accroissement considérable du nombre de formations associées au cours de la période 1979-1983, qui sont passées de 809 unités à 940 unités, ce qui a pour effet d'augmenter l'effectif des chercheurs-équivalents temps plein. Toutefois, la politique de recrutement s'essouffle à partir de 1986. La deuxième source de déséquilibre réside dans la nature même des investissements, qui privilégient nettement les très grands équipements (TGE). Cette tendance s'est nettement affirmée depuis 1976. La structure de la répartition des moyens par modes d'action, qui comprend cinq postes (TGE, actions incitatives, actions d'intervention sur programmes, équipements mi-lourds, soutien de base), révèle le poids croissant des très grands équipements scientifiques, dont la part passe de 19,9\% en 1982 à $26,5 \%$ en 1987.

15 La principale victime de ce double déséquilibre budgétaire est le soutien de base aux laboratoires, défini comme étant la somme des moyens courants en fonctionnement et en équipement moyen, hors infrastructures, hors équipements mi-lourds et actions incitatives. Le rapport entre le soutien de base et l'effectif chercheurs CNRS, exprimé en francs constants, enregistre une baisse de $15 \%$ entre 1982 et 1990. Il n'a cessé en fait de se dégrader depuis les années 1970. L'effort d'investissement lourd a, de même, pour contrepartie une faible croissance du soutien de base des laboratoires: celle-ci s'accélère certes entre 1982-1983 et 1983-1984, s'élevant de $+5 \%$ à $+10,7 \%$, mais pour mieux s'affaiblir au cours des années suivantes. Si l'enveloppe consacrée aux TGE marque une pause à partir de la deuxième moitié des années 1980, il n'en va pas de même de la masse salariale, de nouveau en forte augmentation à partir de 1988. En dépit des orientations et des bonnes résolutions avancées par les schémas directeurs successifs, les finances du CNRS ne semblent pas pouvoir sortir de cette quadrature du cercle.

16 Cette asphyxie progressive des moyens des laboratoires aboutit à un autre déséquilibre au début des années 1990, interne cette fois au budget de l'équipement: les crédits de paiements (CP), qui représentent le montant réel annuel dont disposent les laboratoires pour financer leurs investissements, ne sont plus en mesure de couvrir les autorisations de programmes (AP) qui sont le critère retenu par le CNRS depuis le début des années 1970 pour présenter son budget d'équipement. La différence se traduit en 1994 par un déficit de 550 millions de francs. Face à cette situation de crise, le directeur général, Guy Aubert, impose des mesures drastiques et procède à un audit de l'organisme, confié à l'inspecteur des Finances Jean Charvellin. En contrepartie de sa politique de clarification et de son engagement à rééquilibrer les sources de financement de l'organisme - notamment par un rapprochement accru avec le monde industriel - Guy Aubert arrache une contribution exceptionnelle de l'État. La substantielle augmentation du budget du CNRS en 1995 (+ 18,6\% en francs constants par rapport à 1994) doit donc être interprétée non pas comme l'amorce d'une nouvelle phase de relance financière de l'organisme, mais bien comme une mesure de recapitalisation de ce vaste vaisseau de la recherche pluridisciplinaire qu'est devenu le CNRS. 


\section{Conclusion}

L'étude du budget montre la prodigieuse montée en puissance de l'organisme au cours de la deuxième moitié du XX $\mathrm{XX}^{\mathrm{e}}$ siècle. Du point de vue financier, le CNRS est aussi parvenu à se hisser au cœur de la recherche fondamentale française. Toutefois, le début des années 1970 marque une inflexion dans le rythme de croissance des subventions publiques. Son ralentissement a mis au jour le problème de la masse salariale qui s'était posé dès les années 1950. Masquée pendant les années fastes des budgets de croissance, la part des dépenses en personnel s'avère être une menace sérieuse dans la mesure où l'augmentation des crédits d'équipement n'obéit pas à la même constance. Le vieillissement de la communauté scientifique et technique, ainsi que le poids croissant des très grands équipements, ne font qu'accroître les tensions sur l'équilibre financier du Centre. La rationalisation des choix budgétaires n'a pas empêché une dégradation continuelle de la situation qui aboutit à la diminution drastique des moyens mis à la disposition des laboratoires et à la crise de trésorerie qui sévit à l'aube des années 1990.

\section{NOTES}

1. Cette inflation documentaire est le résultat de l'action engagée par la nouvelle direction administrative et financière qui cherche à comprendre dans ses moindres détails la gestion des deniers publics et du personnel par l'établissement. Pour les sources, cf. note 1 du précédent article : Marnot Bruno, «Le budget du CNRS, I. De la Libération à 1968 ", La revue pour l'histoire du CNRS, nº25, 2010, p. 38-42.

2. Précisons que la notion d'enveloppe recherche n'est plus officiellement retenue dans le budget de 1971, au moment même où le rôle de la DGRST a singulièrement faibli. Il lui est substituée la notion plus large de « crédits soumis à discussion interministérielle ».

3. ITA : ingénieurs, techniciens et administratifs.

4. C'est l'étalon de référence utilisé à partir des années 1980. Le BCRD regroupe le budget du ministère de l'Enseignement supérieur et de la Recherche (MESR), pour plus de la moitié, et les budgets civils des autres ministères.

\section{RÉSUMÉS}

Après avoir abordé l'évolution du budget du CNRS au cours «des années fastes » allant de la Libération à 1968 dans le précédent numéro de cette revue, Bruno Marnot poursuit ici son étude des budgets du Centre sur les trois décennies suivantes. Au cours de cette période, marquée par une conjoncture économique beaucoup moins brillante, les insuffisances budgétaires entraînent 
une importante diminution des moyens mis à la disposition des laboratoires et mènent à la crise de trésorerie qui sévit à l'aube des années 1990.

After discussing, in the previous issue of this journal, the budgetary developments of the CNRS during the "good years", from the Liberation to 1968, Bruno Marnot proceeds here with the study of the Centre budgets all along the next three decades. During this period, marked by more stringent economic conditions, the budgetary inadequacies result in a significant decrease of the laboratory resources and lead to the funds crisis of the early 1990 s.

\section{AUTEUR}

\section{BRUNO MARNOT}

Agrégé et Docteur en Histoire de l'Université Paris IV Sorbonne, Bruno Marnot est actuellement maître de conférences habilité à l'Université Michel de Montaigne Bordeaux III et chargé de cours à l'IEP de Bordeaux. 